\title{
Meniscal Repair and Parameniscal Cyst Excision with Knee Arthroscopic Surgery: A Case Report
}

\author{
Bambang Tutuko', I Gusti Ngurah Wien Aryana \\ ${ }^{1}$ Intern of Orthopaedic and Traumatology Department, Bali Royal Hospital \\ ${ }^{2}$ Consultant of Orthopaedic and Traumatology Department, Bali Royal Hospital \\ Corresponding Author: Bambang Tutuko
}

\begin{abstract}
Background: Meniscal cysts were rare case. There's a 50 to $100 \%$ chance forming cyst to the tear if there was an injury happens. Trauma would cause tears happen in meniscus, leads to formation of hemorrhage which formed mucoid degeneration. The necrosis happens locally plus degeneration of mucoid forming a cyst. Thus, the meniscal cysts challenge the clinician to have clinical reasoning so then the patient can get accurate diagnosis and preferred management.

Method: This paper is a case report of surgery on a patient present with cysts on lateral para meniscus which done arthroscopically with all inside technique.

Results: The procedure which done by arthroscope and motorized shaver had a great outcome in this patient which were analyze from Visual Analog Scale (VAS) and Western Ontario and McMaster Index (WOMAC) 3 months after the procedure, and reach VAS score of 2/10 which was mild pain and 59,8 in WOMAC score.

Conclusion: The arthroscopy and all inside technique with motorized shaver is a choice of lateral para meniscal cysts surgery with good results to be considered by orthopedic surgeons.
\end{abstract}

Keywords: Lateral, Meniscus Tear, Parameniscal cyst, Arthroscopy

\section{INTRODUCTION}

Meniscal cysts were one of rare case which reported incidence ranged $8 \%$ in histologic and imaging studies. With frequent aged report from 20 to 30 years old. Nowadays, many clinician reported that lateral meniscus cysts were more frequent than the medial one although the tears in medial meniscus were more common. ${ }^{1}$ If there any injury happens in knee which torn the meniscus apart, there's a 50 to $100 \%$ chance forming a small cyst to the tear, which it is a response of tissue healing. If the meniscal cysts start to form, it will be the source of discomfort feel in the joint line. $^{2}$ Trauma were one of many injury which cause tears happen in meniscus, it leads to formation of hemorrhage along meniscus which formed mucoid degeneration. ${ }^{3}$
Afterwards, the necrosis happens locally plus degeneration of mucoid forming a cyst. Thus, the trauma would cause the synovial fluid extruded through the tears. Pain would specifically happens in erected position and tend to present with tenderness. If the knee was extended, it would make the cyst more visualable. ${ }^{4}$ If the cysts were occurs in greater area of meniscus, they were called para meniscal cysts. The cysts of lateral meniscus are about 20 to $60 \%$ were more palpable than the medial one which only $6 \% .^{5}$

Thus, the meniscal cysts might challenge the clinician to have clinical reasoning set of skills so then the patient can get accurate diagnosis and preferred management. Differential diagnose of cyst were cyst of synovial, ganglion cyst in ACL and PCL, osteophytes, proximal tibiofibular 

report.

joint cysts and traumatic bursitis. ${ }^{2}$ As of much differential diagnosis that there is, the clinician would have to analyze the patient history through complete anamnesis, physical examination, imaging results and intervention action due to test the suspected diagnoses. The authors present a case report which shown a patient present with cysts on lateral para meniscus which describe the imaging results and management and form a proper diagnosis for the patient.

\section{CASE PRESENTATION}

21 years old female came with chief complaint of pain and swelled of inner aspect of right knee. She also had history of trauma three weeks ago, it happens when she slip off from the stairs which forced her right knee to support her body weight. Afterwards, the pain and swelling start to form, in physical examination was seen a swelling in knee joint line with firm consistency. She also being tested positive for McMurray test. The patient came with severe (6/10) grade pain with Visual Analog Scale (VAS). Patient also felt hard to flex her right knee.

From imaging results, a plain x-ray of right knee was taken with anteroposterior and lateral views showed soft tissue swelling at the region around the lateral femoral condyle and the clinician ordered MRI examination which pictures a partial tear of the posterolateral meniscus from posterior-oblique aspect and anterolateral meniscus from horizontal aspect with observation of lateral parameniscal cyst of right knee, there's also pictured minimal effusion of retro patellar burst and sprained lateral ligamentum collateral (figure 1). This patient also examined with knee scoring of Western Ontario and McMaster Index (WOMAC), the total score which based on patient history was 59,8 .

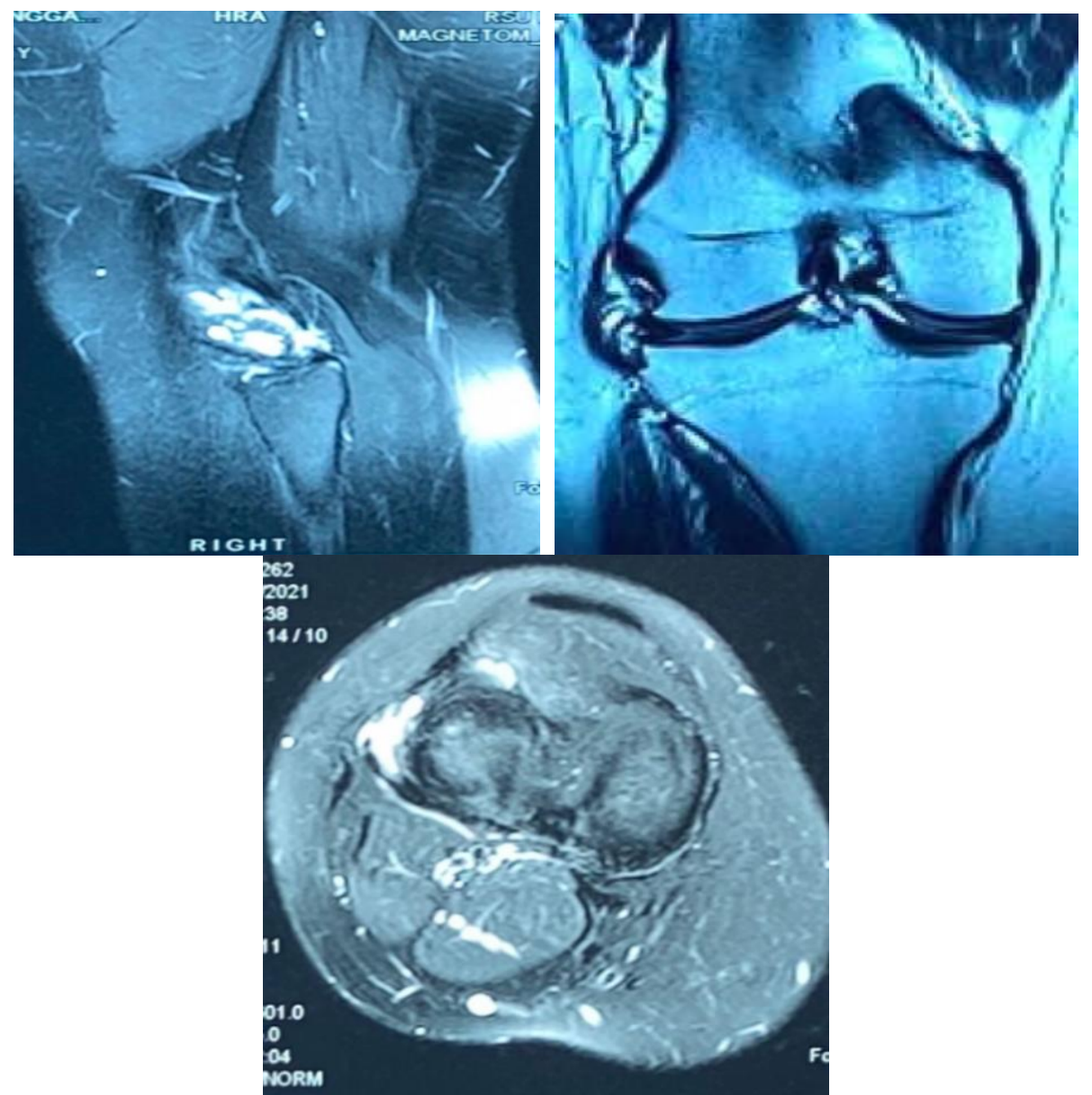

Figure 1. MRI examination shows a partial tear of the posterolateral and anterolateral meniscus with observation of lateral parameniscal cyst of right knee. 


\section{PROCEDURE}

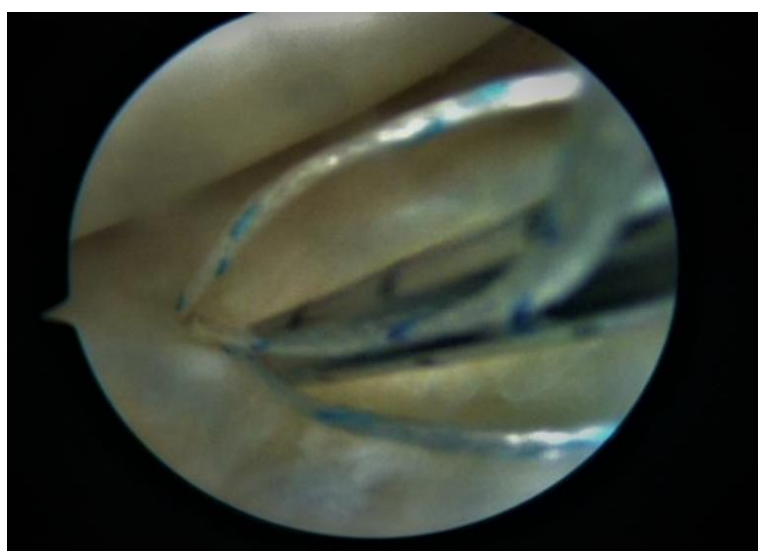

Figure 2. Arthroscopy view which shown the meniscal repair with all inside technique

The procedure was done with anesthesia and the patient were posted for arthroscopy to repair the lateral meniscus tear with all inside technique and to repair the inside-out anterior horn (figure 2). The anesthesia technique was done with spinal anesthesia which the patient was positioned in supine also with $90^{\circ}$ flexed knee. Port of entry was made then afterward shown that anterior and posterior cruciate ligaments were intact, and so does medial meniscus. A rod with sheath was inserted in to the septum, and the arthroscope was then entered through anteromedial portal. Arthroscope and instruments were easy to switch through the port of entry.

Viewed from anteromedial port of entry, the cyst then resected by motorized shaver (figure 3). The patients VAS were then analyze 3 months after the procedure, and reach score of $2 / 10$ which was mild pain. The pain was relieved and the movement of the right knee was normal. The follow up then observed for 1 month and there is no evidence of recurrence of pain and swelling. Also the WOMAC score observe 3 months after the surgery which indicated magnificent result with score of 81,8 .

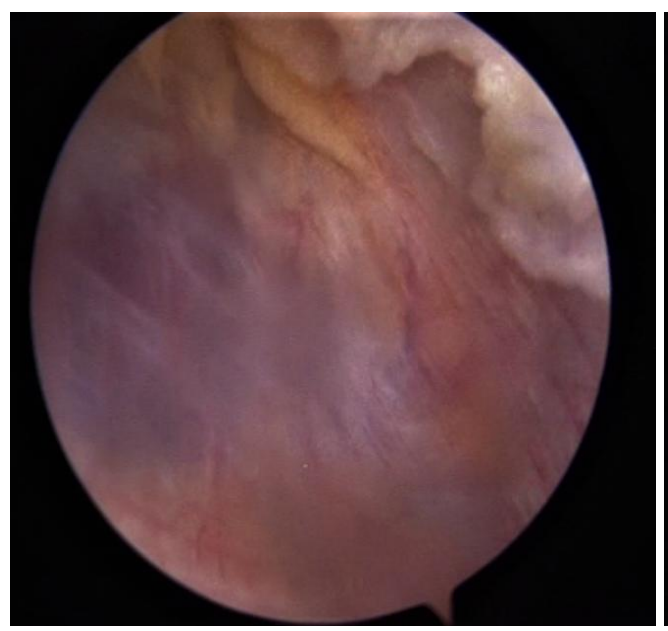

Figure 3. The two pictures shown the parameniscal cyst from the arthroscopic view

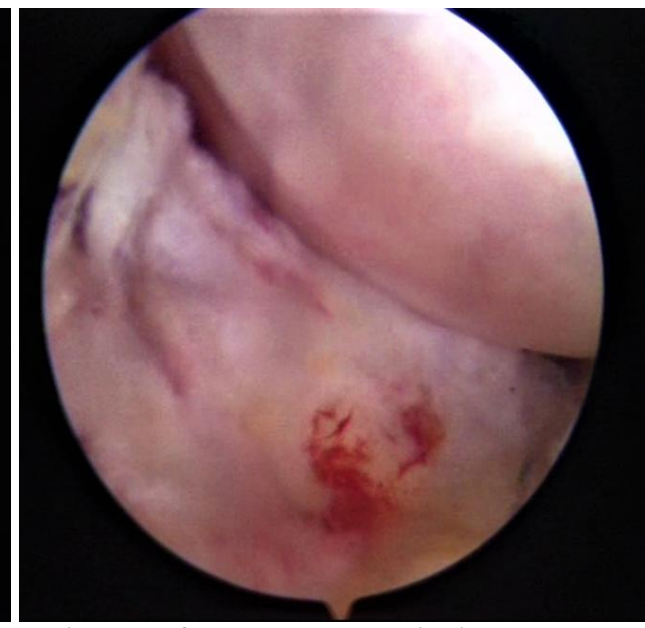

tear of the posterolateral meniscus from posterior-oblique aspect and anterolateral meniscus from horizontal aspect with observation of lateral parameniscal cyst of right knee, there's also pictured minimal effusion of retro patellar burst and sprained lateral ligamentum collateral.

Nowadays, the clinician believes that trauma was the cause of meniscal tears in adult with 20 to 30 years old. The trauma would lead to formation of hemorrhage along meniscus which formed mucoid 
degeneration. ${ }^{3,7}$ The patient was a 21 years old female came with chief complaint of pain and swelling on her right knee. She had history of trauma three weeks ago, it happens when she slip off from the stairs which forced her right knee to support her body weight.

The physical examination also done in patient who suspected with meniscal tears, few types of examination can be done by the clinician in order to get the specific type of injury happen in knee. Such examination like Apley grind test, Bochler test, Thessaly test and McMurray which had 77 to $98 \%$ specificity to detect meniscus injury. ${ }^{8}$ In these patient, the McMurray test was done with full flexion of her right knee to $90^{\circ}$ with full tibial internal rotation and then with full tibial external rotation, the results produces "click" sound in association with torn meniscus and the patient felt so painful.

The pain VAS also done in these patient, the VAS itself was a measurement of pain which widely used in injury of musculoskeletal, it is an unidimensional measure which provided from the patient complaint of pain, which range from "no pain", mild pain until severe pain, where they place pointer in the VAS diagram according to the pain they felt. In musculoskeletal injury, the validity of VAS measurement has shown to be highly correlated with the verbal description of "nil", "mild", "moderate", "severe" and "very severe". 9 In this patient, the VAS scale was 6/10 which determined the pain which the patient felt was severe. The technique done in this patient was combined technique of all inside for lateral meniscus tear and inside out for anterior horn repair, which in other case reports with lateral meniscus tear patient, the combined technique gave $60 \%$ to $80 \%$ success rate for isolated meniscal repairs and $85 \%$ to $90 \%$ when performed in reconstructed anterior cruciate ligament. $^{10}$

In the other parameniscal cyst case report, the technique used for surgery was using arthroscopic for lateral parameniscal cyst decompression, the technique was useful for visualize the entire cyst, and such technique would provide easy instrument handling. ${ }^{11}$ The technique surgery which us done is this patient also using arthroscopy which we inserted through anteromedial portal. Arthroscope and instruments were easy to switch through the port of entry. Viewed from anteromedial port of entry, the cyst then resected by motorized shaver.

In other studies also reported the good outcome from this surgery technique which examined 105 patient with lateral meniscal cyst from arthroscopic treatment, measured with visual analog scale, where $71 \%$ of patient presented with final VAS below 3/10, and $29 \%$ patient shown 4-6/10 VAS score with recurrence cyst. $^{12}$ This patient which first had 6/10 VAS score where it termed as "severe pain" had got a good outcome with the result of 2/10 VAS score after 3 months follow up regarding the patient self scored.

In other study, which used WOMAC score for quality of life instruments in meniscal tear patients, with 18 months post surgery reported that 55 patients had a good recovery outcome with total improved score above $50 \%{ }^{13}$ In this patient, we also examined her with the WOMAC score which from the questionnaire of pain, stiffness and pain in daily life scored 59,8. After 3 months post surgery, the patient then done the WOMAC test which gave a good improvement of 81,8 .

\section{CONCLUSION}

The lateral parameniscal case was one of the rare cases happen in adult which was needed experience set of skill to determine the history, a proper diagnosis and a proper surgery technique. In patient in this case, had to do the all inside with arthroscopic diagnosing, which gave the patient the good outcome of relieve pain and swelling with no pictures of recurrence.

\section{Acknowledgement: None}



report.

\section{Funding}

The authors received no financial support for the research, authorship, and/or publication of this article.

\section{Disclosure of Interest}

The authors declare that they have no conflicts of interest concerning this article.

\section{REFERENCES}

1. Bhandari H, Venkatachalam K. A case of para meniscal cyst of knee managed arthroscopically. SAJB. 2017; 5: 303-305.

2. Crowell MS, Westrick RB, Fogarty BT. Cysts of the lateral meniscus. Int J Sports Phys Ther 2013; 8: 340-348.

3. Herode P, Shroff A, Mandlewala V, et al. Post-traumatic Medial Meniscal Cyst in young: A case report. J Orthop case reports 2016; 6: 7-9.

4. Ohishi T, Torikai E, Suzuki D, et al. Arthroscopic treatment of a medial meniscal cyst using a posterior trans-septal approach: A case report. Sport Med Arthrosc Rehabil Ther Technol 2010; 2: 1-5.

5. Chen H. Diagnosis and Treatment of a Lateral Meniscal Cyst with Musculoskeletal Ultrasound. Case Rep Orthop 2015; 2015: $1-3$.

6. Kim SH, Lee H, Jang Y, et al. Diagnostic Accuracy of Magnetic Resonance Imaging in the Detection of Type and Location of Meniscus Tears: Comparison with Arthroscopic Findings. J Clin Med. 2021 Feb; 10(4): 606. doi: 10.3390/jcm10040606
7. Wesdorp MA, Eijgenraam SM, Meuffels DE, et al. Traumatic Meniscal Tears Are Associated With Meniscal Degeneration. Am J Sports Med 2020; 48: 2345-2352.

8. Evans PJ, Bell GD, Frank C. Prospective evaluation of the McMurray test. Am $J$ Sports Med 1993; 21: 604-608.

9. Hawker GA, Mian S, Kendzerska T, et al. Measures of adult pain: Visual Analog Scale for Pain (VAS Pain), Numeric Rating Scale for Pain (NRS Pain), McGill Pain Questionnaire (MPQ), Short-Form McGill Pain Questionnaire (SF-MPQ), Chronic Pain Grade Scale (CPGS), Short Form-36 Bodily Pain Scale (SF. Arthritis Care Res 2011; 63: 240-252.

10. Nelson CG, Bonner KF. Inside-Out Meniscus Repair. Arthrosc Tech 2013; 2: e453-e460.

11. Haklar U, Ayhan E, Ustundag S, et al. A new arthroscopic technique for lateral parameniscal cyst decompression. Knee 2014; 21: 126-128.

12. Hulet C, Souquet D, Alexandre P, et al. Arthroscopic treatment of 105 lateral meniscal cysts with 5-year average followup. Arthroscopy 2004; 20: 831-836.

13. Sgroi M, Kocak $S$, Reichel $H$, et al. Comparison of 3 Knee-Specific Quality-ofLife Instruments for Patients With Meniscal Tears. Orthop J Sport Med 2018; 6: 1-6.

How to cite this article: Tutuko B, I Gusti Ngurah Wien Aryana. Meniscal repair and parameniscal cyst excision with knee arthroscopic surgery: a case report. Int J Health Sci Res. 2021; 11(10): 172-176. DOI: https:// doi.org/10.52403/ijhsr.20211022 